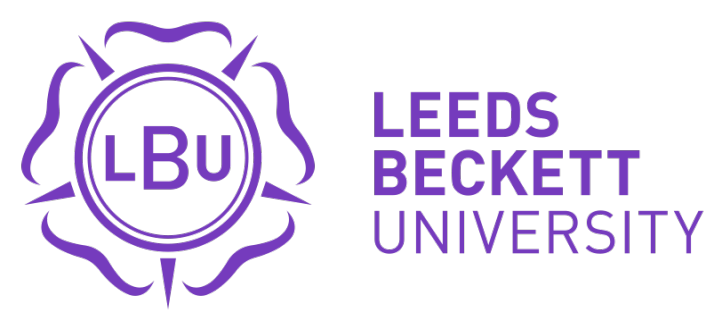

Citation:

Hind, $\mathrm{K}$ and Torgerson, $\mathrm{D}$ and McKenna, $\mathrm{J}$ and Ashby, $\mathrm{R}$ and Daly-Smith, $\mathrm{A}$ and Truscott, $\mathrm{J}$ and MacKay, $\mathrm{H}$ and Jennings, A (2014) Developing Interventions for Children's Exercise (DICE): a pilot evaluation of school-based exercise interventions for primary school children aged 7 to 8 years. Journal of physical activity \& health, 11 (4). 699 - 704. ISSN 1543-3080 DOI: https://doi.org/10.1123/jpah.2012-0116

Link to Leeds Beckett Repository record:

https://eprints.leedsbeckett.ac.uk/id/eprint/238/

Document Version:

Article (Updated Version)

The aim of the Leeds Beckett Repository is to provide open access to our research, as required by funder policies and permitted by publishers and copyright law.

The Leeds Beckett repository holds a wide range of publications, each of which has been checked for copyright and the relevant embargo period has been applied by the Research Services team.

We operate on a standard take-down policy. If you are the author or publisher of an output and you would like it removed from the repository, please contact us and we will investigate on a case-by-case basis.

Each thesis in the repository has been cleared where necessary by the author for third party copyright. If you would like a thesis to be removed from the repository or believe there is an issue with copyright, please contact us on openaccess@leedsbeckett.ac.uk and we will investigate on a case-by-case basis. 


\title{
Developing Interventions for Children's Exercise (D.I.C.E.): A pilot evaluation of school-based exercise interventions for primary school children aged 7 to 8 years
}

Hind K, Torgerson D, McKenna J, Ashby R, Daley-Smith A, Truscott JG, MacKay H.

\author{
E: k.hind@leedsmet.ac.uk
}

\begin{abstract}
Background: Developing Interventions for Children's Exercise (DICE) is an initiative aimed at determining effective school-based exercise programmes. In order to assess feasibility, we conducted a pilot study of exercise sessions which varied in duration and frequency.

Methods: Exercise interventions were delivered to Year 3 pupils (age 7 to 8 years; $n=73$ ) in primary schools within Yorkshire, UK. Evaluations were conducted using focus group sessions, questionnaires and observations.

Results: The study revealed positive aspects of all interventions, including favourable effects on children's concentration during lessons and identified the value of incorporation of the DICE concept into curriculum lessons. Children appeared enthused and reported well-being and enjoyment. Areas requiring attention were the need for appropriate timetabling of sessions and ensuring the availability of space.

Conclusion: The concept and sessions were well-accepted by teachers who confirmed their full support of any future implementation There appears to be potential for the encouragement and empowerment of teachers to support physical activity and healthy school environments, and to take an interest in the health of their pupils. Ultimately, these findings should assist in the design of successful exercise interventions in the school setting.
\end{abstract}




\section{Introduction}

Despite the robust evidence for the key role of physical activity in the primary prevention of diseases such as osteoporosis and cardiovascular disease $(1,2)$, there is concern that a large majority of children are not participating in the recommended amount of physical activity for health (3). The Chief Medical Officer (CMO) of England recommended that children and young people should include activities to improve bone health at least twice a week and achieve a total of at least 60 minutes of at least moderate intensity physical activity each day (4). Yet, in the Avon Longitudinal Study of Parents and Children (ALSPAC), which is tracking 14,000 children from birth, only $2.5 \%$ of girls and boys aged 11 years attained these recommended levels (3).

The World Health Organisation (5) identified schools as a target setting for the promotion of physical activity among children. The school setting is a unique environment for children to learn and practice healthy behaviours $(5,6)$, not least because most children are enrolled at a school where they spend almost half of their waking hours there. Although in most schools physical education (PE) is part of the curriculum, PE lessons can occur infrequently and children are relatively inactive in them (7). For this reason, specific physical activity interventions have been encouraged (8). This notion is supported by a recent Cochrane review of school-based exercise interventions, which recommended ongoing physical activity promotion within schools (8).

Developing Interventions for Children's Exercise (DICE) is a research initiative that has been designed with the primary aim to maximise bone health in children, through pragmatic, school-based weight-bearing exercise. DICE interventions are designed to ensure that they are low cost and can be delivered within the school environment using existing facilities. Consistent with the action plan of the Health Protection Agency (9), DICE also helps to explore the potential responsiveness and effectiveness of teachers and teaching assistants to deliver these interventions.

Elsewhere, there have been a number of school-based intervention studies which have demonstrated significant benefits to the health of children. These health benefits include increasing bone mineral content (10), reducing blood pressure (11), increasing lean muscle mass (12), and increasing aerobic capacity (13). Most studies have reported teachers as the primary intervention provider, with research staff and volunteers as additional providers $(8,10-13)$. Although school-based exercise interventions for research (such as improving bone accrual) have been implemented to varying degrees of success elsewhere, much of this work has beendelivered outside of the UK and has not primarily focused upon acceptance of the interventions among teachers and children (10-17). The purpose of this pilot study was to evaluate the implementation, acceptance and feasibility of DICE exercise sessions within the primary school setting within the UK, with a view to conducting a full randomised controlled trial investigating the optimal school-based exercise programme for improving bone accrual in primary school children. 


\section{Methods}

Seventy three Year 3 students (age 7 to 8 years) and their class teachers, from three primary schools within West Yorkshire, participated in this pilot intervention trial between October 2010 and February 2011. The socio-economic areas of the schools were determined by postcode and area socio-economic status according to household income, educational attainment, skill levels, and concerns about crime, antisocial behaviour and availability of facilities (18). All children who were able to take part in physical education lessons were invited to participate. Subsequently, there were no children who were unable to take part. In total 73 pupils (39 females) participated (14 from School 1, 30 from School 2 and 29 in School 3). The study was reviewed and approved by the Research Ethics Committee from Leeds Metropolitan University. Informed consent was provided by teachers and the parents of the children. Additionally, assent was provided by the children. All researchers who visited the schools were cleared by the Criminal Records Bureau (CRB) and were experienced in working with children within a school setting.

The pilot study was conducted in each school and in total, lasted 5 weeks (this included 1 week for teacher preparation, 3 weeks for the exercise interventions, and 1 week for evaluation). The first week was dedicated to intervention preparation which involved meetings with teachers and introductions to the children. The exercise interventions are described in Table 1 . The interventions were primarily aimed at improving bone accretion and bone strength, in addition to overall physical fitness.

Therefore each intervention involved weight-bearing exercises such as jumping, skipping and dancing which were performed to music. There were a variety of exercises from which the research assistant and the teachers could choose from (Table 1). The concept of the first intervention, 'Bounce at the Bell' was taken from a successful study in Canada which incorporated regular 3 minute bursts of jumping exercises into the school day, and which was carried out in the classroom (14). The landing magnitude of each exercise session was approximately 1 to $5 \mathrm{x}$ body weight (17). Children changed into gym shoes for Intervention 2 and into full PE kit for Intervention 3. A sports-specialist research assistant (RA) led the first day of exercise sessions for each intervention week, and the teachers led the proceeding sessions. The protocol for delivery was as follows:

Monday - Delivery by the RA with class teacher observing. Wednesday - Delivery by the classteacher with RA observing. Friday - Delivery by the class teacher. No observation.

Evaluation: The majority of the evaluative measures were qualitative contributions from teachers, pupils and the RA. Data were collected via three methods: 1) one-to-one interviews with the class teacher, 2) focus groups with the children and 3) researcher observations. All schools participated in the three data-producing parts of the evaluation. 
The teachers of each participating class were interviewed by the RA once, and these interviews were conducted during the week following the close of the exercise interventions. Teacher interviews were conducted away from the class and without any input from the children. The interviews were included as part of the evaluation to establish teacher perceptions of the exercise interventions. Teachers were especially invited to consider how the exercise interventions fitted within the school day, to articulate specific problems and difficulties, to note any influences on pupil motivation, behaviour and concentration and to offer suggestions about preferred session length. These themes were then used to analyse the resulting transcripts which were prepared by the RA.

Focus group sessions were conducted with the children during the week following the close of the exercise interventions. There was one focus group session per class. In order to enable the children to criticise then project comfortably, the focus group sessions were informal and conducted without the class teacher. The focus group sessions provided the children with the opportunity to convey their perspectives on the sessions. Although the focus group sessions were shaped around a set of questions, a flexible approach was adopted to allow discussions to develop and to further enable the children to participate comfortably. Children were asked to identify favourite exercises, things they did not enjoy, their physical feelings and feelings of well-being during and after the exercises, their thoughts about the music, and if they had any ideas for exercises which they would like to do. Focus groups involved informal discussions with the RA and questions with shows of hands to quantify responses. As with teacher interviews; the broad themes of the discussion provided the framework for developing the final analysis.

The RA observations also contributed to the evaluation of the interventions.Summary notes were made following each week of the exercise interventions to include perceptions on whether or not the RA believed the sessions had been accepted and enjoyed, and if there had been any problems or difficulties. Written field notes were also made by the RA during the teacher-led exercise sessions. These notes emphasised perceived enjoyment, pupil inclusion, engagement in the sessions, and the quality of teachers' delivery of the session. The observational data were used as supplementary data within the evaluation.

Data analysis: The results from the teacher interviews, focus group sessions and RA observations were qualitatively evaluated by the Principal Researcher, and when there were any uncertainties within the notes, the RA was consulted. In order to perform clear and appropriate evaluations, results were analysed under separate headings: perceptions on each of the exercise sessions; preferred and least preferred exercise sessions (and reasons why); issues and problems; positive aspects; acceptability and feasibility. 


\section{Results}

Teacher perceptions: Varying views from teachers were provided regarding the $4 \times 5$ minute exercise session. For School 1 it represented a problem with fitting in with scheduled use of existing space: '...difficult to access the hall and could not use the classroom because of art work'.

In contrast, the teacher from School 2, felt that the 4 x 5 minute sessions, '...fitted in really well', going on to say that this was their preferred intervention. The teacher from School 3 found benefits with the short exercise session, '...really well liked by the pupils and we believe that short burst activity improved their concentration'.

The 2 x 10 minute exercise sessions were well accepted by School 1, '...the sessions fitted in easy with the school day and provided a sustained and energetic work-out for the children'. This was repeated by the teacher from School 3, '...these sessions worked well... we jumped to the beat of the clock which helped the children keep count, and to a beat, which is useful for music lessons'. In contrast, the teacher from School 2 found that '...these were the most difficult sessions in respect of staff to deliver'. Here the explanation was that although the classroom was large enough to accommodate the $4 \times 5$ minute exercise sessions, it '...proved too small for the 10-minute sessions and therefore the school hall had to be used'.

In School 1 the $1 \times 20$ minute session was found to be '... more difficult to incorporate', while the teacher from School 2 stated that these sessions were '...quite difficult in terms of incorporation into the school day and may be disruptive ...seemed long', yet still noted that 'the kids really liked the circuits and the length of the exercises'. The teacher from School 3, who was a PE specialist, preferred the longer session both personally and for the children, '... I really liked the longer session. The kids really enjoyed the circuit-based activities... from a teaching perspective the 20-minute sessions worked best for us'.

Teachers were asked to identify any particular issues with the exercise interventions. With School 2 the main issue was the incorporation of the longer exercises into the school day, as discussed above. In School 1, the teacher commented that there were no specific problems, 'the children really enjoyed the exercises and were able to complete them without any difficulties. There were no participation issues; the sessions actually helped participation for one of our children'. The teacher from School 3 provided a similar response, 'Importantly, none! The pupils loved all of the exercises and really engaged with them'.

Perceptions were also provided on aspects of the exercises that were found to be difficult. In School 1, the teacher stated, '...some children found that the dance routines were a little tricky, but this could be overcome with practice and improved co-ordination'. In School 2 the teacher reported no difficulties 
in terms of the aspects of the exercises, although commented that the 20-minute session seemed long. The teacher from School 3 stated that there were no difficulties, although, '...access to the hall is not always easy to get’.

Comments regarding particularly positive aspects of the exercises were recorded. Benefits in concentration and enjoyment were reported by all 3 teachers. In School 1, '...The children seemed to concentrate in class more after the sessions and were happier and more alert' and, '...[sessions were] really well liked by the pupils and as well as an improvement in fitness, we believe that shortburst activity improved their concentration'. In School 2, '...All of the children in the class engaged with the exercises and the study. During the science lessons we would always look at how the exercises we were doing were beneficial'. Enthusiasm for the DICE programme was such that it was being adopted during PE lessons by School 3, 'We are going to continue to use the music and theme for jumping for warm-ups before PE lessons'.

Children's perceptions on the exercise sessions: All children reported that they enjoyed all of the exercise interventions (100\%). Children from School 1 particularly enjoyed the sessions when a dance sequence was incorporated into the bouncing. As an indication of its creative, educational, potential, some children asked if they could make up their own routines using jumps, skips and stretches. In all three schools, the children's favourite exercises were jumping, dancing and the use of ladders for hopping. In School 3, the children also reported that they enjoyed the circuits.

When asked if they found anything difficult about the exercises one pupil from School 1, said they found the longer exercises 'difficult to keep going'. Yet, once they were able to keep going, there were comments from the children that they felt more energetic having done the sessions. In School 2, only 3 girls reported any kind of difficulty; they found the 20 minute session more physically demanding. Two children from School 3 found that skipping was the most difficult part of the sessions. In terms of wellness, all children said that they felt better after the exercises. Other comments by children from School 3 were, '...We have got strong muscles in our legs now', '...we feel healthy and want to do more' and one asked '...Can we do it every day?'

Researcher observations: The RA reported in the field notes that the children and teachers were fully engaged with each of the exercise sessions. The children all appeared to enjoy exercising to the music. The children liked to perform a sequence of exercises which they could practice and learn. The field notes also indicated that the teachers appeared to be very enthusiastic about both the research and the effects of the sessions on their pupils. Two schools have already incorporated the basis of the DICE research into science lessons, and one school has requested that the RA assist with one of their science lessons during which the focus was on the overall effect of exercise on the body. The RA reported no problems with motivation in any of the observed sessions in any school. Filed notes from 
School 1, noted that the exercise sessions encouraged the participation of one child who would otherwise have sat alone and not joined in. This child had learning difficulties and, according to the teacher, found it difficult to socialise. At first, he was very quiet and withdrawn. He observed the class taking part in the first 5 minute exercise session, and then 'helped' with the second session by clapping to the music. By the third session he joined in for a few of the exercises and by the final 5minute session of the day he was joining in fully and enjoying himself. These findings showed how class teachers can quickly handle the issues that may cause concern among some pupils.

Can children perform the exercises? With reference to the RA field notes, all children appeared to cope with the exercise sessions. The skipping sessions were difficult for some children, especially among those who had never skipped before. Some children observed that their legs had ached in the days following initial sessions, but this was not reported negatively; they were using this to show their interest in, and commitment to, this new programme.

Can teachers deliver the exercises? Teachers led the sessions on the Wednesday and Friday and they reported no specific problems around the delivery. Initially, teachers were not always confident about taking the sessions and felt that this would have been resolved with a small amount of in-service training. In School 2, the teacher seemed to find the exercises more physically tiring than the younger teacher in School 1 who 'really enjoyed the work-out' and the PE specialist in School 3. The teachers - 2 of whom were non-PE specialists -were delighted that the children were experiencing structured and developmentally-appropriate exercise.

\section{Discussion}

The school setting is frequently identified as the ideal setting for physical activity promotion interventions $(4,5,8)$. This pilot study revealed positive aspects of three school-based interventions, as well as highlighting areas that require particular attention for the programme to work effectively. Importantly, teachers were positive about the whole experience. This enthusiasm spanned the delivery of exercise sessions, through to their facilitation of the focus group meetings and completion of the evaluation. Every child participated, which offers an indication of their acceptability among boys and girls aged 7 and 8 . Whilst we do not claim that our findings are representative of every potential school which may embark on similar physical activity programmes, the study informs on future intervention design.

The sessions were designed to be incorporated into the school day, and previous research suggested that this should not detrimentally influence academic performance (8). Far from offering a neutral 'academic effect', we found that the interventions had a positive effect on teacher-reported pupil 
concentration during class time. Indeed, there is growing evidence that regular exercise may enhance cognitive performance in children $(19,20)$.

Children noted a number of important positive experiences arising from DICE exposure. Firstly, no child and no parent declined to participate. A 100\% participation rate for any programme is rare, but bodes well for the potential generalisation of outcomes. For similar reasons, it was also helpful that the participating children were from a diffuse range of socio-economic (SES) areas. Children from lower socioeconomic backgrounds generally show the lowest levels of physical activity (20), meaning that the $100 \%$ engagement with these communities is especially important. DICE appears to be able to realise the potential for schools to influence community well-being.

Children reported few difficulties and these were associated with skipping and general fitness. As a strategy to minimise difficulties, we suggest that prior to the introduction of physical activity interventions such as DICE, children are taught basic skills that can help with more the complex activities such as skipping with ropes. This could be incorporated into PE lessons or as DICE preparation sessions. Children could also be encouraged to practice at home and should be reminded that with regular practice, comes improvement in skills and in general fitness. These values could also be taught in lessons about healthy living/healthy body.

Socially, the exercise sessions provided opportunities to integrate children from different ethnic groups, which were particularly diverse in School 1. Social inclusion through exercising together as a whole class brought enjoyment and benefits for the children, including children who may otherwise be withdrawn in class. This was observed in School 1. Structured exercising as a class may also heighten individual self-efficacy for exercise, and perhaps even elevate levels of more generalized self-esteem (19). Teachers and pupils' contributions and field notes all indicated excellent pupil engagement with the exercises and they were motivated both to participate and continue their involvement. Even where the exercises took time to learn, as in skipping, the pupils found sufficient reason to continue trying so that their skills improved. These positive learning effects - which rely on persistence, delay of gratification and an array of other features that indicate cognitive as well as physical development (20) - were also found to have positive effects on participants' self-reported feelings of well-being. This was noted by both teachers and pupils. Exercise to music was enthusiastically received by all of the children. The use of 'world' music to motivate and refine engagement in DICE activities could also contribute to cross-curricular delivery, particularly in lessons on geography and culture.

One major challenge in improving children's health through physical activity is establishing the sustainability of the interventions. Research to date indicates that success and adherence to schoolbased exercise interventions can be improved by using multi-component approaches in children (6). 
One strategy has been to include families as important mediators for exercise outside the school and for positive attitudes towards exercise in general. Van Sluijs et al (21) found strong evidence for the inclusion of family components, and it has been suggested that this may be more applicable to younger children than adolescents who are trying to become autonomous (6). Thus, the involvement of a family component may promote long term feasibility of the DICE protocol. In economic terms, the DICE protocol was designed to require little financing and to make use of school's current resources, thereby aiming to be cost effective. In terms of the practicality of the interventions, we suggest that feasibility for long term adherence could be enhanced by ensuring that the exercise programmes advance over time and include new exercises and perhaps awards, to engage the students. This may be more difficult for the first intervention (of 5 minute bouts) due to the constraints of time and space. In this case, the introduction of hand-held equipment such as bands and dumbbells could be included, and enable other regions of the body to be exercised. It should also be considered that children progress through school and change classes on a yearly basis. This could offer opportunities for interventions to be tailored to age and developed ability.

We confirm the numerous benefits of implementing a school-based physical activity intervention. However, one of the main issues highlighted by the teachers in this study is to ensure that sessions can be accommodated during the routines of busy school timetables. Whilst teachers were accepting of the interventions, there were comments that the $4 \times 5$ minute sessions (School 1) and the 2x10 minute sessions (School 2) were sometimes difficult to accommodate, mainly due the restricted availability of already-limited school space. For example, the $4 \times 5$ minute sessions are designed to take place in the classroom. At the time of the pilot School 1 had art-work on the classroom floor, meaning that the class needed to relocate to complete the sessions in the school hall. School 2 also had problems accessing the school hall for the 2x10minute sessions. One strategy to help combat these problems could be to ensure that rooms are organised well in advance to secure the delivery of the exercise sessions. Attention should also be given to ensuring that the necessary space is available for exercise delivery. To this end, realistic space assessments should be conducted. The support of Head teachers and Year 3 teachers will be important here, as will meetings and consultations to agree on the best times during the school day to incorporate the intervention. In schools with limited space, specific strategies could be adopted to facilitate the exercise protocols. Safety procedures should be established during the first couple of sessions to include rules on entering and exiting the space, use of equipment, stop and start cues, warm up and cool down routines, transitions and emergency situations/fire drills. The safe performance of the exercise sessions in limited space, such as a classroom may provide an opportunity to teach children how to stay active despite a limited environment and limited resources. Other concepts could include going outside to exercise whenever possible, if exercising inside, always make sure that objects are moved out of the way so as not to be hazardous, and always have a plan B. This strategy should not only improve overall teacher acceptance but also their facilitation of the exercise sessions. 
Theteam identified that compliance and acceptability may be improved through the use of a website showing details of the exercise sessions and supportive teaching resources. The teaching resources would relate to the DICE concept and address areas such as strong bones and healthy heart. These resources could be downloaded for incorporation into lessons, and supports statements from teachers that the inclusion of the DICE concept into lessons could benefit teaching (for example, in science lessons). Since it is widely recognized that teachers are more strongly motivated by curricular-related issues than health-related issues (22), this may help to prompt and encourage teachers whose motivation for continued involvement may wane.

In developing any exercise intervention it is necessary to establish both the duration and frequency of the sessions. Three different DICE interventions were 'road tested' in this pilot study. The first consisted short bursts of exercise interspersed throughout the school day, the second comprised two relatively short sessions per day and the third, one longer session per day. Although each intervention amounts to 20 minutes of exercise (three times per week), they may represent different levels of classroom 'disruption', which can be compounded by space issues in respective schools. Where space is problematic, and given that children also regularly receive (i) PE lessons, (ii) other periodic movement opportunities throughout the day, and bearing in mind the children's age and the findings from this pilot study; a total of 20 minutes of DICE activities using these venues would offer a viable alternative. It is also possible that longer sessions will be more disruptive to the school day and be less likely to be maintained.

Given the importance of delivering interventions through existing staffing, and to fit with existing policy directions (23), the development and establishment of teacher confidence is vital. The DICE exercise protocols appeared to be well accepted by the teachers and only one of these teachers were specialists in PE. For PE specialist primary teachers, the DICE protocol may be attractive in that they could use the activities to complement children's learning in PE about how and why exercise is important for health, and to develop skills in movement, coordination, and sequences (Key Stage 1 and 2 objectives). The protocols were not too complicated and used simple exercises to music, with minimal equipment, therefore should be attractive to teachers who are not specialised in PE. In order to improve compliance from teachers of all professional backgrounds, we also suggest that at least three preliminary dedicated training sessions are provided in preparation for the interventions. This could be supplemented with top-up training as the programmes progresses and with supporting activity cards. These cards could act as prompts and provide further information to support teachers, both within the DICE sessions but also to develop cross-curricular themes. These cards may also be used to develop aid pupil leadership by involving them in supervising parts of the DICE session, thereby reducing any burden on the teacher. This would concur with NICE guidance on physical 
activity interventions for children, by involving them directly and encouraging confident exercising (23).

This pilot evaluation was limited by its non-randomised and relatively small sample. In this, it is not possible to generalise findings to other schools and students. We also recognise that the short duration over which the interventions were conducted may not be sufficient to identify any possible problems that could appear if the intervention were conducted over a longer period of time. Furthermore, permanent acceptance of the interventions used in this study cannot be inferred due the short duration. In this respect, it is possible that the acceptance and enjoyment conveyed by the children simply reflects a response to something new and different. With these limitations in mind, we suggest that longer term implementation of the interventions may provide further important information.

In conclusion, this pilot study indicated that children were interested and complied with the interventions, and all parents provided consent for their children to participate. Teachers indicated that the exercises could be readily incorporated into the school day. There were notable, acute benefits of involvement for the children - sessions appeared to promote inclusion, self-reported feelings of wellbeing and of heightened concentration throughout the school day. All children appeared fully engaged with the exercises and study and all reported enjoyment, although this finding, and the aforementioned, should be confirmed through longer implementation of the interventions. In light of our evaluation, we make the following recommendations:

1. From the outset, involve teachers and Heads in carefully timetabling and committing to delivering sessions.

2. Confirm that sufficient space is available for the class to fully participate in the sessions.

3. Develop and refine teacher confidence for delivering exercise sessions.

4. Provide additional teaching resources/support materials so teachers can link the DICE exercises to other areas of teaching and learning.

5. Focus group sessions may be a valuable method to engage pupils in evaluations of schoolbased interventions.

Acknowledgements: With sincere thanks to all children, teachers and schools who took part in this study so enthusiastically. 


\section{References}

1. Hind K, Burrows M (2005) Weight-bearing exercise for bone mineral accrual in children and adolescents: a systematic review of controlled trials. Bone

2. Aadahl M, von Hoth Saiths L, Pisinger C (2009) Five year change in physical activity is associated with changes in cardiovascular disease risk factors: the Inter99 study. Prevent Med 48:326-330

3. Riddoch CJ, Mattocks C, Deere K, Saunders J, Kirkby J, Tilling K, Leary SD, Blair SN, Ness AR (2007) Objective measurement of levels and patterns of physical activity.', Arch Dis Child 92: 963-639

4. Chief Medical Officers Report 2004 http://www.dh.gov.uk/en/Publicationsandstatistics/ Publications/PublicationsPolicyAndGuidance/DH_4080994 accessed on the 12th January, 2011

5. World Health Organisation (2004) Young peoples' health in context. Health Policy for Children and Adolescents, No 4.

6. Kriemler S, MeyerU, Martin E (2011) Effect of school-based interventions on physical activity and fitness in children and adolescents: a review of reviews and systematic update. $B$ J Sports Med 45:923-928

7. MacKenzie TL, Feldman H, Woods SE (1995) Children's activity levels and lesson context during third grade physical education. Res Quart Exerc Sport 66:184-193

8. Dobbins M, DeCaby K, Robertson A (2009) School-based physical activity programmes the for promotion of physical activity and fitness in children and adolescents aged 6 - 18 (Review) The Cochrane Library. Issue 4

9. Health Protection Agency (2009) A Children's Environment and Health Strategy for the UK inhttp://www.hpa.org.uk/web/HPAwebFile/HPAweb_C/1237889522947

10. Linden C, Ahlborg HG, Besjakau J (2006) A school curriculum-based exercise program increases bone mineral accrual and bone size in prepubertal girls: 2 year data from the Pediatric Osteoporosis Prevention (POP) study. J Bone Mineral Res21:829-833

11. Hansen HS, Froberg K, Hyldebrandt N (1991) A controlled study of 8 months of physical training and reduction of blood pressure in children: the Odense Childhood Study BMJ 303:682-685

12. MacKelvie KJ, MacKay HA, Khan KM, Crocker PRE (2001) A school-based exercise intervention augments bone mineral accrual in early pubertal girls. J Pediatr 139:501-508

13. Carrel AL, Clark RR, Peterson SE (2005) Improvement of fitness, body composition and insulin sensitivity in overweight children in a school based exercise program: a randomised controlled study. Arch Pediatr Adolesc Med 159:963-968

14. McKay HA, Petit MA, Schutz RW, Prior JC, Barr SI, Khan KM (2009) Augmented trochanteric bone mineral density after modified physical education classes: a randomised 
school-based exercise intervention study in prepubescent and early pubescent children. $J$ Pediatr 136: 156-162

15. Fuchs RK, Bauer JJ, Snow C (2001) Jumping improves hip and lumbar spine bone mass in prepuberscent children: a randomised controlled trial. J Bone Miner Res 16: 148-156

16. Petit MA, McKay HA, MacKelvie KJ, Heinonen A, Khan KM, Beck TJ (2002) A randomised school-based jumping intervention confers site and maturity-specific benefits on one or more structural properties in girls: a hip structural analysis study. J Bone Miner Res 17: 363-372

17. McKay HA, MacLean L, Petit M, MacKelvie O’Brien K, Janssen P, Beck T, Khan KM (2005) 'Bounce at the bell': a novel program of short bouts of exercise improves proximal femur bone mass in early pubertal children. Br J Sports Med39: 521-526

18. South Leeds Programme 2007, http://www.leeds.gov.uk/files/2007/week28/inter 12f9c034e051-480d-b71f-ea5f5d54e0be_7ecfc51e-8906-49a7-859c-125c85f52e79 Accessed on $12^{\text {th }}$ January 2011

19. Hill AP, King NA, Armstrong TP (2007) The contribution of physical activity and sedentary behaviour to the growth and development of children and adolescents. Implications for overweight and obesity. Sports Med 37:533-545

20. Taras H (2005) Physical activity and student performance at school. J Sch Health 75: 214-218

21. Van Sluisj EMF, McMinn AM, Griffin S (2007) Effectiveness of interventions to promotephysical activity in children and adolescents: a systematic review of controlled trials. BMJ 335:703

22. Jourdan D, Mannix McNamara P, Simar C, Geary T, Pommier J (2010) Factors influencing the contribution of staff to health education in schools. Health Educ. Res 25: 519-530

23. National Institute for Health and Clinical Excellence (NICE) (2009) Promoting physical activity, active play and sport for pre-school and school-age children, and young people in family, pre-school, school and community settings. NICE Public Health Guidance 17 
Table 1. DICE exercise interventions

\begin{tabular}{|c|c|c|}
\hline $\begin{array}{c}\text { Frequency } \\
\text { (Mon, Wed and Fri) }\end{array}$ & $\begin{array}{c}\text { Exercises } \\
\text { (magnitude } 1-5 \mathrm{x} \text { body weight) }\end{array}$ & $\mathbf{n}$ \\
\hline $\begin{array}{l}\text { Week } 1 \\
4 \text { x } 5 \text { minutes per day } \\
\text { (triggered by morning, lunch- } \\
\text { time and end of school bells) } \\
\text { Classroom-based; to music }\end{array}$ & $\begin{array}{l}\text { Bounce at the Bell } \\
\text { Jogging on-the-spot, small on-the-spot jumps and } \\
\text { skips, hopping and } 5 \text { counter movement jumps }\end{array}$ & $\begin{array}{c}73 \\
\text { (3 schools) }\end{array}$ \\
\hline $\begin{array}{l}\text { Week } 2 \\
2 \text { x } 10 \text { minutes per day (during } \\
\text { morning and afternoon break } \\
\text { times) } \\
\text { Hall or outside (jumps and } \\
\text { circuit training to music) }\end{array}$ & $\begin{array}{l}\text { Break-time Bounce } \\
\text { *Jumps - jumping jacks, high knees, side to side } \\
\text { jumps, drop jumps [progressing } 10-30 \mathrm{~cm} \text { ] } \\
\text { *Circuit training ( } 5 \text { stations, including counter } \\
\text { movement jumps, side to side, drop jumps } \\
\text { [progressing } 10-50 \mathrm{~cm} \text { ] and use of ladders) } \\
\text { *Team games- wheel barrows, sack races, all fours } \\
\text { run, piggy backs, hopping, jumping }\end{array}$ & $\begin{array}{c}73 \\
\text { (3 schools) }\end{array}$ \\
\hline $\begin{array}{l}\text { Week } 3 \\
1 \text { x } 20 \text { minutes per day (at } \\
\text { lunch-time) } \\
\text { Hall or outside (jumps and } \\
\text { circuit training to music) }\end{array}$ & $\begin{array}{l}\text { Jump to the Beat, Salsa Skip or Disco Circuits } \\
\text { *Jumping jacks, high knees, side-to-side jumps, } \\
\text { drop jump }[10 \mathrm{~cm}] \\
\text { *Skipping } \\
\text { *Circuit training (8 stations,including counter } \\
\text { movement jumps, side-to-side jumps, drop jumps } \\
\text { [10cm] and use of ladders) }\end{array}$ & $\begin{array}{c}73 \\
\text { (3 schools) }\end{array}$ \\
\hline
\end{tabular}

